\title{
EFECTO NEGATIVO DEL RIEGO SALINO EN COMPONENTES DEL RENDIMIENTO Y FITOQUÍMICOS DE CHILE (Capsicum annuum) INOCULADO CON HONGOS MICORRÍCICOS ARBUSCULARES
}

\author{
NEGATIVE EFFECT OF SALINE IRRIGATION ON YIELD COMPONENTS \\ AND PHYTOCHEMICALS OF PEPPER (Capsicum annuUm) \\ INOCULATED WITH ARBUSCULAR MYCORRHIZAL FUNGI
}

\author{
Graciela L. Grimaldo-Pantoja', Genhua Niư ${ }^{2}$ Youping Sun ${ }^{2}$, Arturo Castro-Rocha', Emilio \\ Álvarez-Parrilla' ${ }^{1}$, Juan P. Flores-Márgez ${ }^{1}$, Baltazar Corral-Díaz ${ }^{1}$ y Pedro Osuna-Ávila ${ }^{1}$ *
}

\begin{abstract}
'Departamento de Ciencias Químico Biológicas, Instituto de Ciencias Biomédicas, Universidad Autónoma de Ciudad Juárez. Anillo Envolvente y Estocolmo s/n. 32310, Ciudad Juárez, Chihuahua, México. ${ }^{2}$ Texas A\&M AgriLife Research Center at El Paso. 1380 Texas A\&M Circle. 79927-5020, El Paso, Texas, United States, Tel 9158599111

*Autor para correspondencia (posuna@uacj.mx)
\end{abstract}

\section{RESUMEN}

Se evaluó el efecto de dos consorcios de hongos micorrícicos arbusculares (HMA) aislados de plantas de huizache (Acacia sp.) (HMA1) y de una asociación entre Larrea tridentata y Opuntia sp. (HMA2), nativas del desierto Chihuahuense, en los componentes del rendimiento y fitoquímicos en plantas de chile Jalapeño var. $M$ crecidas bajo estrés salino en condiciones de invernadero. El diseño experimental constó de dos factores: HMA (HMA-, HMA1

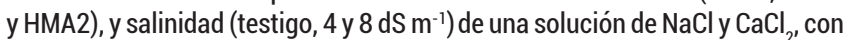
nueve repeticiones por tratamiento. El número de esporas fue contabilizado y las plantas se inocularon con 40,000 esporas. La solución salina se aplicó 30 $\mathrm{d}$ después de la inoculación durante ocho semanas. El fósforo se aplicó con una solución de $44 \mathrm{mg} \mathrm{L}^{-1}$ de $\mathrm{KH}_{2} \mathrm{PO}_{4}$ a las plantas sin inocular y de $22 \mathrm{mg} \mathrm{L}^{-1}$ a las plantas inoculadas. La micorrización al final del experimento osciló entre 22 y $32 \%$ en las plantas inoculadas. La salinidad afectó significativamente el desarrollo y el verdor de las plantas. Aunque el factor HMA tuvo un efecto negativo en el número de frutos, no se afectó el rendimiento en el peso fresco de los frutos. La salinidad tuvo un efecto negativo en el peso fresco de los frutos. Los HMA incrementaron significativamente el contenido de fósforo y ácido ascórbico, sin verse afectados el desarrollo y fisiología de la planta a pesar de la reducción en la cantidad de fósforo exógeno aplicado. La concentración de fósforo en los frutos fue mayor en las plantas inoculadas con HMA2. A mayor salinidad, los frutos tuvieron menor concentración de ácido ascórbico pero mayor concentración de fenoles totales en los frutos. El contenido de capsaicinoides no fue afectado por ninguno de los factores estudiados.

Palabras clave: Capsicum annuum, cv. Jalapeño, micorriza, estrés abiótico.

\section{SUMMARY}

The effect of two consortia of arbuscular mycorrhizal fungi (AMF) isolated from huizache (Acacia sp.) plants (HMA1) and an association between Larrea tridentata and Opuntia sp. (HMA2), both native of the Chihuahuan Desert, on the yield components and fruit phytochemical content of Jalapeño var. $M$ pepper plants (Capsicum annuum L.) subjected to saline stress, was evaluated under greenhouse conditions. The experiment had two factors: AMF (HMA-, HMA1 and HMA2) and salinity levels (control, 4 and $8 \mathrm{dS} \mathrm{m}^{-1}$ ) of a $\mathrm{NaCl}$ and $\mathrm{CaCl}_{2}$ solution. Each treatment had nine replications. The number of spores per gram of soil were counted and pepper plants were inoculated with 40,000 spores each. Salinity was applied $30 \mathrm{~d}$ after the inoculation for eight consecutive weeks. Phosphorus was applied with a solution of $44 \mathrm{mg} \mathrm{L}^{-1}$ of $\mathrm{KH}_{2} \mathrm{PO}_{4}$ for non-inoculated plants and $22 \mathrm{mg} \mathrm{L}^{-1}$ for inoculated ones. Colonization at the end of the experiment oscillated between 22 and $32 \%$ in inoculated plants The AMF factor had a negative effect on the number of fruits, however yields were not affected regarding fresh fruit weight. Salinity had a negative effect on fresh fruit weight. AMF significantly increased the content of phosphorus and ascorbic acid, without affecting the development and physiology of the plant in spite of the reduced amount of exogenous phosphorus applied. Phosphorus concentration was higher in plants inoculated with HMA2. The factors salinity and AMF had a significant effect on the concentration of phenols and ascorbic acid in the fruits. Higher salinity decreased the concentration of ascorbic acid in fruits but increased the concentration of total phenols. The content of capsaicinoids was not affected by any of the factors.

Index words: Capsicum annuum, cv. Jalapeño, mycorrhiza, abiotic stress.

\section{INTRODUCCIÓN}

En la región árida del Noroeste de Chihuahua, la producción de chile (Capsicum annum L.) es una de las principales actividades económicas (SIAP, 2015). La salinidad es un factor ambiental limitante para el crecimiento de las plantas. Las plantas de chile son sensibles a este estrés que afecta su producción (Azuma et al., 2010). Altas concentraciones de sal en el suelo y agua reducen la absorción de agua y nutrientes e inhiben procesos metabólicos y afecta la captación de nutrientes. Una alternativa que puede ayudar a las plantas contra este tipo de estrés es el uso de hongos micorrícicos arbusculares (HMA) (Kumar et al., 2015). Los HMA se asocian con más de $80 \%$ de las plantas terrestres (Smith y Read, 2008). Esta asociación mutualista ayuda a mejorar la captación de fósforo y agua e incrementa la tolerancia contra estrés biótico y abiótico (Sharif y Claassen, 2011). 
En plantas de chile (C. annum L. cv. California Wonder $300)$ se ha evaluado Rhizophagus intraradices en diferentes concentraciones de $\mathrm{NaCl}(0,50,100$ y $200 \mathrm{mM})$. Las plantas del tratamiento testigo tenían más contenido de sodio en los tejidos; y la biomasa de la parte aérea y de la raíz fue mayor en las plantas inoculadas con el hongo. En ambos tratamientos se observó un incremento en la cantidad de prolina a mayor salinidad (Beltrano et al., 2013). Otro estudio realizado en C. annum cv. 11B 14 evaluó el efecto de Glomus clarum en condiciones de alta salinidad. Se detectó que la salinidad disminuyó la concentración de fósforo, nitrógeno y potasio en plantas tratadas, así como en tallo y raíz; sin embargo, en las plantas inoculadas con el HMA el contenido de los nutrientes fue igual al de las plantas sin sal y se redujo la pérdida de electrolitos a través de la membrana celular (Kaya et al., 2009).

Existe limitada información sobre la planta de chile en respuesta a la salinidad y no hay estudios de la inoculación con HMA del Desierto Chihuahuense en chile Jalapeño M que se cultiva en la región. El objetivo fue evaluar el efecto de estos microorganismos sobre los componentes del rendimiento y cantidad de fitoquímicos en los frutos verdes de chile Jalapeño en condiciones de riego salino en invernadero y durante tres cosechas.

\section{MATERIALES Y MÉTODOS}

\section{Material fúngico}

Consorcios de HMA fueron recolectados en febrero del 2014 en el Desierto Chihuahuense a orillas del condado de El Paso, Texas. Los consorcios fueron recolectados de rizosfera de huizache (Acacia sp.) (HMA1) y de una asociación entre Larrea tridentata y Opuntia sp. (HMA2). Las esporas de los consorcios de HMA se propagaron a través de Sorghum sp. y Triticum sp. como plantas trampa en macetas de $8 \mathrm{~L}$ llenadas con mezcla de rizosfera y arena estéril en una proporción 1:1.

Las plantas se desarrollaron durante tres meses (febrero - abril del 2014) en condiciones de invernadero, fertilizadas semanalmente con $200 \mathrm{~mL}$ de solución nutritiva Long Ashton modificada a $22 \mathrm{mg} \mathrm{L}^{-1}$ de fosfato de potasio monobásico $\left(\mathrm{KH}_{2} \mathrm{PO}_{4}\right)$. Pasados los tres meses se recuperó el suelo de las plantas trampa. Se tomaron $50 \mathrm{~g}$ de suelo como muestra para la extracción de esporas mediante el método de Gerdemann y Nicolson (1963) y se contabilizó el número de esporas por gramo de suelo con ayuda de un microscopio estereoscópico (Nikon® SMZ-800). La identificación de las especies de HMA de cada consorcio se postergó hasta verificar su potencial biotecnológico.

\section{Material vegetal}

Semillas de chile (Capsicum annuum L., var. Jalapeño M) se pusieron a germinar, el 2 de mayo del 2014, en celdas de plástico negro de 72 pozos rellenadas con sustrato Metro Mix 360 (SunGro® Horticulture., Bellevue, WA, USA). Plántulas de $20 \mathrm{~d}$ se trasplantaron a macetas de $7.5 \mathrm{~L}$ donde se usó el mismo sustrato. Según el tratamiento, las plantas se inocularon con el mismo número de esporas $(40,000$ esporas por planta) de HMA1 o HMA2.

Para regular el fósforo (P), las plantas se fertilizaron cada dos semanas con $1 \mathrm{~g}$ de fertilizante South West Green Maker el cual carece de P y contiene 8.9 \% de nitrógeno en forma de amoniaco, $9.1 \%$ de nitrógeno en forma de urea, $10 \%$ de azufre, $6 \%$ de potasio soluble, $0.02 \%$ de boro, 0.05 $\%$ de cobre, $5 \%$ de hierro, $0.05 \%$ de manganeso y $0.05 \%$ de zinc. Para evitar que las plantas fueran afectadas por deficiencia de $\mathrm{P}$, se agregaron $400 \mathrm{~mL}$ de $\mathrm{KH}_{2} \mathrm{PO}_{4}$ una vez por semana, a una concentración de $44 \mathrm{mg} \mathrm{L}^{-1}$ a las plantas sin inocular y de $22 \mathrm{mg} \mathrm{L}^{-1}$ a las plantas inoculadas con HMA, según los lineamientos establecidos por Davies Jr. et al. (2000).

\section{Tratamientos}

Nueve tratamientos (dos factores con tres niveles cada uno) con nueve repeticiones por tratamiento $(n=81)$ fueron empleados para el presente estudio. Para inducir los niveles de salinidad en los riegos se aplicaron semanalmente soluciones de cloruro de sodio $(\mathrm{NaCl})$ y cloruro de calcio $\left(\mathrm{CaCl}_{2}\right)$ en proporción molar de 2:1 y con niveles de conductividad eléctrica (CE) de 4.1 y 8.1. como fue descrito por Niu et al. (2012). El regado diario se hizo con agua tratada con osmosis inversa. La solución salina se aplicó $30 \mathrm{~d}$ después de que las plantas fueran inoculadas con sus respectivos consorcios de HMA durante ocho semanas consecutivas. Se monitoreó la conductividad eléctrica (CE) y el pH de la solución del sustrato. Las temperaturas en el invernadero fueron de $29.7 \pm 5.3^{\circ} \mathrm{C}$ durante el día y $23.9 \pm$ $3.9^{\circ} \mathrm{C}$ durante la noche. La cantidad de luz fotosintéticamente activa en el invernadero fue de16.2 $\pm 3.4 \mathrm{~mol} \mathrm{~m}^{-2} \mathrm{~d}^{-1}$.

En cada tratamiento se midieron las variables altura, número de hojas totales, área foliar (medida con un medidor de área Li-Cor LI-3100C $\AA$, Lincoln, NE, USA), diámetro del tallo, peso fresco y seco de la parte aérea y de la raíz, longitud de la raíz, antes de comenzar a tratar las plantas con el riego salino y al final del experimento.

\section{Colonización micorrícica}

La colonización micorrícica se calculó antes y después de aplicar los riegos salinos mediante el método de 
Phillips y Hayman (1970). Raíces de $1 \mathrm{~cm}$ se tiñeron con azul de tripano. Se montaron 10 segmentos por portaobjetos con nueve laminillas por tratamiento y se observaron a 40x mediante un microscopio de campo claro (Nikon® SMZ-800). Cada segmento se categorizó con la siguiente escala: 1 (0 \% de colonización), 2 (5 \% de colonización), 3 (30 \% de colonización), 4 (70 \% de colonización), 5 (95\% de colonización). Para calcular la intensidad micorrícica se utilizó la siguiente fórmula (Covacevich et al., 2001):

$$
M \%=\frac{(n 1+5 n 2+30 n 3+70 n 4+95 n 5)}{N}
$$

Donde $\mathrm{N}$ es el número total de fragmentos observados por tratamiento, y n1, n2, n3, n4 y n5 corresponden al número de raíces que se observaron al utilizar la escala.

\section{Variables fisiológicas}

SPAD. El verdor de las hojas se midió con un medidor SPAD (Minolta SPAD 502 Plus Camera Co., Osaka, Japan). Se cuantificó antes de aplicar los riegos salinos y en las semanas dos, cuatro, seis y ocho, durante los riegos salinos, en la tercera y cuarta hojas sanas.

Intercambio de gases. La fotosíntesis neta $\left(P_{n}\right)$ fue medida antes de aplicar los riegos salinos y en la semana dos, cuatro, seis y ocho de tratamiento, con un sistema portátil de fotosíntesis CIRAS-2 (PP Systems, Amesbury, MA, USA) con celda automática universal PLC6 para hoja ancha. Esta variable se midió en la tercera hoja totalmente expandida. Las condiciones ambientales de la celda se mantuvieron a una temperatura de $25^{\circ} \mathrm{C}$ con un flujo de fotones fotosintéticos (PPF) de $1000 \mu \mathrm{mol} \mathrm{m} \mathrm{m}^{-2} \mathrm{~s}^{-1}$ y una concentración de dióxido de carbono $\left(\mathrm{CO}_{2}\right)$ de $375 \mu \mathrm{mol} \mathrm{mol}{ }^{-1}$. Los datos se colectaron cuando las condiciones ambientales y los parámetros del intercambio de gases de la celda fueron estables y días soleados entre las 10:00 y las 12:00 horas en plantas previamente irrigadas.

\section{Cosecha de los frutos}

Debido a que el chile es una hortaliza que se puede cosechar múltiples veces, se realizaron tres cortes durante el experimento en las semanas cinco, siete y ocho de tratamiento. Se cuantificó el número y peso fresco de frutos por planta. Para la cuantificación de fósforo y fitoquímicos sólo se utilizaron frutos sanos de más de $4 \mathrm{~cm}$ de largo.

\section{Contenido de P}

Un gramo de chile seco se incineró a $300^{\circ} \mathrm{C}$ en una mufla (Fisher Thermolyne ${ }^{\mathrm{TM}}$, USA) durante $3 \mathrm{~h}$. A las cenizas se les agregó $1 \mathrm{~mL}$ de ácido clorhídrico $(\mathrm{HCl})$ a $37 \%$ y 19 $\mathrm{mL}$ de agua destilada. La solución se filtró con papel filtro
Whatman \# 42. A $100 \mu \mathrm{L}$ del filtrado se añadió 2,4-dinitrofenol para ajustar el pH. Luego se añadió $5 \mathrm{~mL}$ de ácido cloromolíbdico y se aforó a $25 \mathrm{~mL}$ con agua destilada. Para finalizar se agregaron $0.05 \mathrm{~mL}$ de cloruro estañoso y se incubó por $5 \mathrm{~min}$. La absorbancia se midió a $660 \mathrm{~nm}$ en un espectrofotómetro Genesys 20 (Thermo Spectronic ${ }^{\text {TM }}$, USA) (Jackson, 1982).

\section{Fitoquímicos}

Se cuantificaron: ácido ascórbico, fenoles totales y capsaicinoides en los frutos. Los frutos se cortaron en rodajas, se congelaron a $-80{ }^{\circ} \mathrm{C}$ y se liofilizaron por $3 \mathrm{~d}$ en un liofilizador (Labconco ${ }^{\circledR}$ freeze dryer / shell freeze system, Labconco Corp., Kansas City, MO, USA). Las muestras liofilizadas fueron homogenizadas y almacenadas a $-80{ }^{\circ} \mathrm{C}$ hasta su análisis.

Ácido ascórbico (AA). A $0.1 \mathrm{~g}$ de chiles liofilizados se agregó $2.5 \mathrm{~mL}$ de ácido metafosfórico a $5 \%$ y se sonicó en oscuridad durante 20 min. La mezcla se centrifugó a 1780 $X \mathrm{~g}$ durante 10 min y se colectó el sobrenadante. El sobrenadante se mezcló con $200 \mu \mathrm{L}$ de ácido tricloroacético (TCA) a $6.65 \%$ y $75 \mu \mathrm{L}$ de 2,4-dinitrofenilhidracina (DNPH), y se incubó a $37^{\circ} \mathrm{C}$ por $3 \mathrm{~h}$. Se agregó $0.05 \mathrm{~mL}$ de ácido sulfúrico a $65 \%$. La absorbancia se midió a 520 nm en un lector de microplacas xMark ${ }^{\circledR}$ (Bio-Rad, Berkeley, CA, USA) con ácido ascórbico como estándar y los resultados se expresaron como mg de AA por cada $100 \mathrm{~g}$ de peso seco (González-Aguilar et al., 2008; Oboh y Rocha, 2007).

Fenoles totales. Dos gramos de chile liofilizado se mezclaron con $0.5 \mathrm{~mL}$ de metanol a $80 \%$ y se sonicaron en oscuridad durante $30 \mathrm{~min}$. La mezcla se centrifugó a $1525 \mathrm{Xg}$ por $30 \mathrm{~min}$ y se colectó el sobrenadante, el procedimiento se repitió y los sobrenadantes se mezclaron. Para remover el etanol, el sobrenadante se rotovaporó a $55^{\circ} \mathrm{C}$, y se congeló a $-80^{\circ} \mathrm{C}$. Posteriormente se liofilizó durante $48 \mathrm{~h}$.

Para cuantificar, se preparó una disolución de extracto liofilizado y metanol a $80 \%$ a una concentración de $1 \mathrm{mg}$ $\mathrm{mL}^{-1}$. Se tomaron $500 \mu \mathrm{L}$ de disolución y se mezclaron con $2.5 \mathrm{~mL}$ de reactivo de Folin-Ciocalteu (diluido 1:10 con agua destilada) que se dejó en reposo por 2 min. Se agregaron $2 \mathrm{~mL}$ de carbonato de calcio $\left(\mathrm{Na}_{2} \mathrm{CO}_{3}\right)$ a $7.5 \%$, y se incubó a $45^{\circ} \mathrm{C}$ por $15 \mathrm{~min}$. Se dejó enfriar a temperatura ambiente y se midió la absorbancia a 760 nm. Se utilizó como estándar ácido gálico, y los resultados se expresaron como mg equivalentes de ácido gálico (EAG) por cada $100 \mathrm{~g}$ de peso seco (Alvarez-Parrilla et al., 2011).

Capsaicinoides. La extracción y cuantificación de capsaicinoides se llevó a cabo al mezclar chile liofilizado con 10 $\mathrm{mL}$ de metanol grado HPLC (> 99.9\%). La mezcla se sonicó 
20 min y se centrifugó a 1308 X g durante 5 min para colectar el sobrenadante. La extracción se repitió una segunda vez con los residuos y se combinaron los sobrenadantes. La cuantificación se llevó a cabo por cromatografía de líquidos de alta presión (HPLC) con fase móvil isocrática de acetonitrilo - ácido acético 1 \% en proporción 50:50. La absorbancia se leyó a $860 \mathrm{~nm}$ con con capsaicina como estándar, y los resultados se expresaron como mg equivalentes de capsaicina por cada $100 \mathrm{~g}$ de peso seco (AlvarezParrilla et al., 2011).

\section{Análisis estadístico}

El diseño experimental fue factorial con dos factores: HMA (HMA-, HMA1 y HMA2), y riego con tres niveles de salinidad con conductividad eléctrica de 1.4 (testigo), 4.0 y $8.0 \mathrm{dS} \mathrm{m}^{-1}$. En las variables relacionadas con los frutos se consideró el número de cortes como un factor adicional. Los datos se analizaron por medio de un ANOVA factorial a un $a=0.05$, con comparación de medias mediante la prueba de Tukey a un nivel de confianza a $95 \%$ con el programa estadístico SPPS versión 20.0 para Windows.

\section{RESULTADOS Y DISCUSIÓN}

\section{Colonización micorrícica}

La colonización antes de aplicar la salinidad era de 10 $\%$ en los tratamientos inoculados y de $2 \%$ en plantas sin inocular. Al final del experimento las plantas inoculadas con HMA1 presentaron una colonización entre 22 y $32 \%$, mientras que en las plantas inoculadas con HMA2 fue entre 22 y $28 \%$. El factor HMA mostró efecto significativo ( $P$ $<0.05)$, mientras que los niveles de salinidad no afectaron la colonización micorrícica (Cuadro 1). En las plantas sin inocular se observó hasta 10 \% de colonización.

En este tipo de estudios se puede presentar colonización en las plantas sin inocular de hasta $40 \%$ de micorrización (Khan et al., 2014). La colonización micorrícica es afectada cuando se aplica estrés salino. En plantas de chile cv. $\mathrm{PKM}_{1}$ inoculadas con $R$. intraradices y tratadas con $\mathrm{NaCl}$ a 25 y $50 \mathrm{mM}$, el porcentaje de colonización disminuyó de 66 a 24 y $15 \%$, respectivamente (Selvakumar y Thamizhiniyan, 2011). Cuando se aplicó solución salina de $\mathrm{NaCl}$ en concentraciones de 100 y $200 \mathrm{mM}$ a plantas de chile (C. annuum California Wonder 300) inoculadas con $R$. intraradices, la colonización se redujo de 80 a 65 y $58 \%$, respectivamente (Beltrano et al., 2013).

\section{Variables de crecimiento}

Después de aplicar durante ocho semanas los niveles de salinidad se observaron diferencias significativas en las medias de la altura de la planta, número de hojas, área foliar, diámetro del tallo, peso fresco y seco de la parte aérea

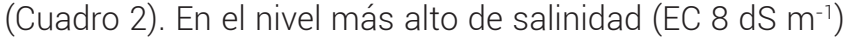
se observó una disminución en las variables antes mencionadas. Esto concuerda con el estudio de Beltrano et al. (2013), quienes reportaron que el contenido de clorofila y el área foliar disminuyeron con la aplicación de solución salina en plantas de chile inoculadas con $R$. intraradices.

Cuadro 1. Porcentaje de colonización micorrícica en chile Jalapeño M antes y después de la aplicación del riego salino.

\begin{tabular}{|c|c|c|}
\hline \multirow{2}{*}{ Tratamiento } & \multicolumn{2}{|c|}{ Colonización micorrícica (\%) } \\
\hline & Antes de los riegos salinos & Después de los riegos salinos \\
\hline & \multicolumn{2}{|c|}{ Testigo } \\
\hline HMA - & $2 a$ & $8.6 \mathrm{bA}$ \\
\hline HMA1 + & $10 \mathrm{a}$ & $22.5 \mathrm{aA}$ \\
\hline \multirow[t]{2}{*}{ HMA2 + } & $10 \mathrm{a}$ & $21.7 \mathrm{aA}$ \\
\hline & \multicolumn{2}{|c|}{ CE $4 \mathrm{dS} \mathrm{m}^{-1}$} \\
\hline HMA - & $2 a$ & $10.0 \mathrm{bA}$ \\
\hline HMA1 + & $10 \mathrm{a}$ & $32.0 \mathrm{aA}$ \\
\hline \multirow[t]{2}{*}{ HMA2 + } & $10 \mathrm{a}$ & 27.9 aA \\
\hline & \multicolumn{2}{|c|}{ CE $8 \mathrm{dS} \mathrm{m}^{-1}$} \\
\hline HMA - & $2 a$ & $9.6 \mathrm{bA}$ \\
\hline HMA1 + & $10 \mathrm{a}$ & $25.8 \mathrm{aA}$ \\
\hline HMA2 + & $10 a$ & $28.7 \mathrm{aA}$ \\
\hline
\end{tabular}

Medias con letras iguales en la misma columna no son estadísticamente diferentes (Tukey, 0.05); las letras en minúscula representan los subgrupos formados por el factor micorriza y las letras mayúsculas por el factor salinidad derivados de la prueba de Tukey; HMA- (plantas testigo); HMA1+ (aislado de Acacia sp.); HMA2+ (aislado de Larrea-Opuntia); CE: conductividad eléctrica. 
En este mismo estudio se observó que el peso seco de la raíz y la parte aérea fue mayor en plantas inoculadas con HMA. Selvakumar y Thamizhiniyan (2011) demostraron que la aplicación de sal disminuyó el peso seco de plantas de chile cv. PKM, sin afectar la altura.

SPAD e intercambio de gases. Los factores salinidad, HMA y tiempo afectaron significativamente las medias del verdor relativo de las plantas (SPAD) (Cuadro 3). Los valores SPAD disminuyeron con los tratamientos de salinidad, y los valores más bajos se registraron en plantas tratadas con la salinidad más alta. Los valores también disminuyeron con el tiempo de exposición a la sal. Díaz et al. (2013) reportaron que, en plantas sin riego salino, las lecturas del SPAD incrementaron significativamente en plantas inoculadas con HMA con respecto a plantas no inoculadas. Según Niu et al. (2010), la salinidad no afectó el contenido relativo de clorofila en hojas de plantas de chile sin inocular con HMA. En intercambio de gases no se detectaron diferencias significativas.

Frutos. Los factores HMA y cosechas tuvieron un efecto

Cuadro 2. Medias de las variables fenológicas de chile Jalapeño M inoculadas con HMA en condiciones de estrés salino durante 8 semanas.

\begin{tabular}{|c|c|c|c|c|c|c|}
\hline Tratamiento & $\mathrm{NH}$ & Área foliar $\left(\mathrm{cm}^{2}\right)$ & Altura (cm) & DT (mm) & $\mathrm{PF}(\mathrm{g})$ & PS (g) \\
\hline & \multicolumn{6}{|c|}{ Testigo } \\
\hline HMA - & 364 a & $5376.34 a b$ & $87.36 \mathrm{a}$ & $17.87 \mathrm{ab}$ & $370.7 b$ & $31.7 \mathrm{a}$ \\
\hline HMA1 + & 332 a & $5540.52 \mathrm{ab}$ & $89.74 \mathrm{a}$ & $15.23 a b$ & $397.1 \mathrm{~b}$ & $34.1 \mathrm{a}$ \\
\hline \multirow[t]{2}{*}{ HMA2 + } & 349 a & $5335.62 a b$ & 87.43 a & $15.17 \mathrm{ab}$ & $369.7 b$ & $30.8 \mathrm{a}$ \\
\hline & \multicolumn{6}{|c|}{$\mathrm{CE} 4 \mathrm{dS} \mathrm{m}^{-1}$} \\
\hline HMA - & 298 a & 5623.73 a & $84.81 \mathrm{a}$ & $15.42 \mathrm{a}$ & 396.6 a & $29.3 b$ \\
\hline HMA1 + & 323 a & $5814.90 \mathrm{a}$ & 89.99 a & $14.19 \mathrm{a}$ & $420.2 \mathrm{a}$ & $30.5 b$ \\
\hline \multirow[t]{2}{*}{ HMA2 + } & $330 \mathrm{a}$ & $5812.12 \mathrm{a}$ & $86.76 \mathrm{a}$ & $15.69 \mathrm{a}$ & $411.1 \mathrm{a}$ & $30.3 b$ \\
\hline & \multicolumn{6}{|c|}{$\mathrm{CE} 8 \mathrm{dS} \mathrm{m}^{-1}$} \\
\hline HMA - & $256 b$ & 5164.07 b & $78.60 \mathrm{~b}$ & $14.52 b$ & $382.0 \mathrm{~b}$ & $27.9 c$ \\
\hline HMA1 + & $227 b$ & $4897.11 b$ & $80.86 b$ & $14.09 b$ & $346.9 b$ & $25.0 \mathrm{c}$ \\
\hline HMA2 + & $255 b$ & $4939.64 b$ & $78.80 \mathrm{~b}$ & $14.94 b$ & $354.0 \mathrm{~b}$ & $25.9 c$ \\
\hline
\end{tabular}

Medias sin letras o con letras iguales en la misma columna no son estadísticamente diferentes (Tukey, 0.05); las letras en minúscula representan los subgrupos formados por el factor salinidad derivados de la prueba de Tukey; HMA- (plantas testigo); HMA1+ (aislado de Acacia sp.); HMA2+ (aislado de Larrea-Opuntia); NH: número de hojas; DT: diámetro del tallo; PF: peso fresco de la parte aérea; PS: peso seco de la parte aérea; CE: conductividad eléctrica.

Cuadro 3. Efecto de la salinidad en el verdor (SPAD) en hojas de chile Jalapeño M.

\begin{tabular}{|c|c|c|c|}
\hline Tratamiento & 2 semanas con salinidad & 4 semanas con salinidad & 8 semanas con salinidad \\
\hline & \multicolumn{3}{|c|}{ Testigo } \\
\hline HMA - & 59.6 a & $56.2 \mathrm{a}$ & $40.6 \mathrm{a}$ \\
\hline HMA1 + & 59.6 a & $56.8 \mathrm{a}$ & $46.1 \mathrm{a}$ \\
\hline \multirow[t]{2}{*}{ HMA2 + } & $59.3 \mathrm{a}$ & 55.7 a & $40.4 \mathrm{a}$ \\
\hline & \multicolumn{3}{|c|}{ CE $4 \mathrm{dS} \mathrm{m}^{-1}$} \\
\hline HMA - & $58 b$ & $54 \mathrm{~b}$ & $39.0 \mathrm{~b}$ \\
\hline HMA1 + & $60 b$ & $54.5 b$ & $42.9 \mathrm{~b}$ \\
\hline \multirow[t]{2}{*}{ HMA2 + } & $56.8 b$ & $54.7 b$ & $40.3 b$ \\
\hline & \multicolumn{3}{|c|}{ CE $8 \mathrm{dS} \mathrm{m}^{-1}$} \\
\hline HMA - & $54.5 c$ & $55.3 \mathrm{c}$ & $32.5 c$ \\
\hline HMA1 + & $54.9 c$ & $55.2 \mathrm{c}$ & $33.4 \mathrm{c}$ \\
\hline HMA2 + & $55.6 c$ & $53.9 c$ & $37 c$ \\
\hline
\end{tabular}

Medias sin letras o con letras iguales en la misma columna no son estadísticamente diferentes (Tukey, 0.05); las letras en minúscula representan los subgrupos formados por el factor salinidad derivados de la prueba de Tukey HMA- (plantas testigo); HMA1+ (aislado de Acacia sp.); HMA2+ (aislado de Larrea-Opuntia); CE: conductividad eléctrica. 
significativo en el número de frutos. El número fue disminuyendo con los cortes. En el primer corte se obtuvieron hasta 70 frutos en plantas sin HMA lo cual no presentó diferencias significativas con respecto a la media de 61 frutos por planta de las plantas inoculadas con el consorcio HMA2. Las plantas inoculadas con el consorcio HMA1 fructificaron significativamente menos que las plantas sin inocular, con una media de 55 frutos por planta. En el último corte, el número de frutos disminuyó considerablemente (Cuadro 4).

El peso del fruto fue afectado por el nivel más alto de salinidad y por el número de cortes, ya que en la última cosecha los frutos pesaron menos (Cuadro 4). Plantas inoculadas con HMA tuvieron el mismo peso fresco de frutos que plantas sin inocular, aún, y cuando las plantas sin inocular produjeron más frutos; por lo tanto, los rendimientos no se vieron afectados a pesar de la disminución en el número de frutos. En plantas de chile Valeria inoculadas con R. intraradices, Díaz et al. (2013) reportaron que los frutos presentaron mayor tamaño y peso. Plantas de chile cv.11B 14 inoculadas con G. clarum disminuyeron su rendimiento al aumentar la concentración de sal (Kaya et al., 2009).

Concentración de P. En las plantas inoculadas con HMA2 se cuantificó la mayor cantidad de P (32 $\left.\mathrm{mg} \mathrm{L}^{-1}\right)$ en la primera cosecha. En la segunda cosecha se observó que las plantas sin inocular presentaron la mayor cantidad de $\mathrm{P}$ (Figura 1). Cabe destacar que las plantas inoculadas fueron fertilizadas con la mitad de $\mathrm{P}$ con respecto a las plantas sin inocular; sin embargo, la concentración en frutos no se disminuyó sino hasta la segunda cosecha. Se desconoce si la cosecha de los frutos interfiere de alguna manera con la fisiología de la relación micorrícica. Según Kaya et al. (2009) la concentración de P en plantas de chile disminuye al incrementar los niveles de $\mathrm{NaCl}$; sin embargo, en plantas inoculadas con G. clarum se incrementó la cantidad de este elemento en los tejidos de las plantas.

Ácido ascórbico (AA). Los tratamientos con mayor cantidad de este compuesto fueron las plantas inoculadas con HMA1 y las plantas sin inocular. En cambio, se observó una disminución significativa entre los tratamientos con salinidad. Las plantas tratadas con solución salina de 8 dS $\mathrm{m}^{-1}$ fueron las que presentaron la menor cantidad de AA. A través de las cosechas se observó que las plantas sin inocular presentaron mayor cantidad de AA en el primer corte, mientras que en el segundo hubo una disminución significativa. La cantidad de AA se incrementó en las plantas inoculadas (Cuadro 5).

Estos resultados concuerdan con los reportados por Azuma et al. (2010), quienes monitorearon la concentración de ácido ascórbico en los frutos durante 21 d en plantas de chile bajo condiciones de estrés salino, y encontraron que durante los primeros $7 \mathrm{~d}$ la concentración de este compuesto incrementó con respecto a las plantas testigo. A partir del día 14 existió una disminución en la concentración, mientras que la cantidad de sodio acumulado aumentó. Se han observado resultados similares en plantas de tomate (Solanum lycopersicum L.) expuestas a sequía en donde la concentración de ácido ascórbico disminuye

Cuadro 4. Número y peso fresco de los frutos observados en las diferentes cosechas en chile Jalapeño $\mathrm{M}$ en condiciones de estrés salino.

\begin{tabular}{|c|c|c|c|c|c|c|}
\hline \multirow{2}{*}{ Tratamiento } & \multicolumn{3}{|c|}{ Número de frutos } & \multicolumn{3}{|c|}{ Peso fresco (g) } \\
\hline & Primera & Segunda & Tercera & Primera & Segunda & Tercera \\
\hline & \multicolumn{6}{|c|}{ Testigo } \\
\hline HMA - & $70 \mathrm{a}$ & $36 a$ & $11 \mathrm{a}$ & $290.8 \mathrm{~A}$ & $157.78 \mathrm{~A}$ & $34 \mathrm{~A}$ \\
\hline HMA1 + & $55 b$ & $30 b$ & $15 b$ & $270.66 \mathrm{~A}$ & $123.04 \mathrm{~A}$ & $51 \mathrm{~A}$ \\
\hline \multirow[t]{2}{*}{ HMA2 + } & $61 \mathrm{ab}$ & $33 a b$ & $16 a b$ & $279.96 \mathrm{~A}$ & $125.96 \mathrm{~A}$ & $57 \mathrm{~A}$ \\
\hline & \multicolumn{6}{|c|}{ CE $4 \mathrm{dS} \mathrm{m}^{-1}$} \\
\hline HMA - & $67 \mathrm{a}$ & $32 \mathrm{a}$ & $8 a$ & 248.92 B & $99.36 \mathrm{~B}$ & $26 \mathrm{~B}$ \\
\hline HMA1 + & $61 \mathrm{~b}$ & $35 b$ & $12 b$ & $195.31 \mathrm{~B}$ & $122.47 \mathrm{~B}$ & $36 \mathrm{~B}$ \\
\hline \multirow[t]{2}{*}{ HMA2 + } & $63 a b$ & $36 a b$ & $9 a b$ & 211.03 B & $111.79 \mathrm{~B}$ & $26 \mathrm{~B}$ \\
\hline & \multicolumn{6}{|c|}{ CE $8 \mathrm{dS} \mathrm{m}^{-1}$} \\
\hline HMA - & $59 a$ & $33 \mathrm{a}$ & $8 a$ & 196.66 C & $85.49 \mathrm{C}$ & $19.1 \mathrm{C}$ \\
\hline HMA1 + & $56 b$ & $30 \mathrm{~b}$ & $7 b$ & $175.83 \mathrm{C}$ & $89.43 \mathrm{C}$ & $16.88 \mathrm{C}$ \\
\hline HMA2 + & $66 a b$ & $27 \mathrm{ab}$ & $11 a b$ & $191.97 \mathrm{C}$ & $75.36 \mathrm{C}$ & $25.62 \mathrm{C}$ \\
\hline
\end{tabular}

Medias sin letras o con letras iguales en la misma columna no son estadísticamente diferentes (Tukey, 0.05); las letras en minúscula representan los subgrupos formados por el factor HMA y las letras mayúsculas por el factor salinidad derivados de la prueba de Tukey HMA- (plantas testigo); HMA1+ (aislado de Acacia sp.); HMA2+ (aislado de Larrea-Opuntia); CE: conductividad eléctrica. 


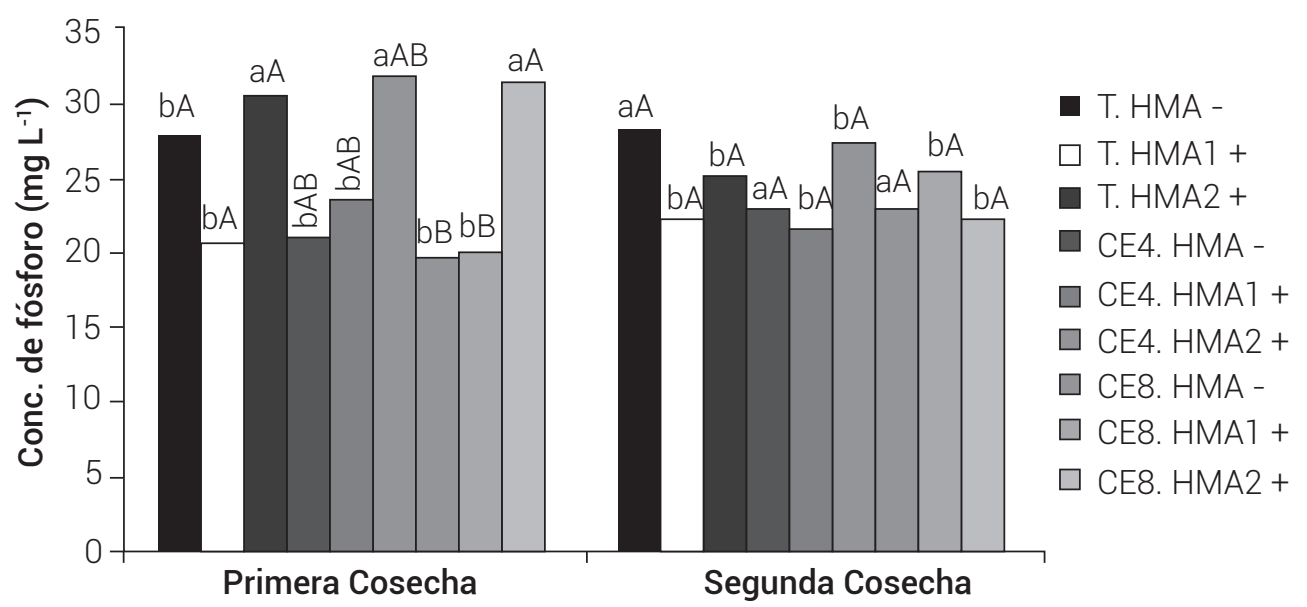

Figura 1. Concentración de fósforo en frutos de chile Jalapeño $M$ en condiciones de estrés salino en las diferentes cosechas. Medias sin letras o con letras iguales en la misma columna no son estadísticamente diferentes (Tukey, 0.05); las letras en minúscula representan los subgrupos formados por el factor HMA y las letras mayúsculas por el factor salinidad derivados de la prueba de Tukey HMA- (plantas testigo); HMA1+ (aislado de Acacia sp.); HMA2+ (aislado de Larrea-Opuntia); CE: conductividad eléctrica.

Cuadro 5. Contenido de fitoquímicos en frutos de chile Jalapeño $M$ en condiciones de estrés salino.

\begin{tabular}{llcccrr}
\hline Cosecha & Tratamiento & AA mgAA/100 g PS & FT mgEAG/100 g PS & Capsaicina $\mu \mathrm{g} / \mathrm{g}$ PS & DHC g/g PS & CT \\
\hline Primera & T. HMA - & $1896.62 \mathrm{aA}$ & $855.52 \mathrm{bC}$ & 3969.05 & 89.08 & 4058.14 \\
& T. HMA1 + & $1404.3 \mathrm{bA}$ & $798.53 \mathrm{cC}$ & 3008.70 & 82.17 & 3090.88 \\
& T. HMA2 + & $1643.32 \mathrm{aA}$ & $825.04 \mathrm{aC}$ & 1445.53 & 65.56 & 1511.09 \\
& CE4. HMA - & $1225.99 \mathrm{aB}$ & $673.7 \mathrm{bB}$ & 3540.10 & 111.03 & 3651.14 \\
& CE4. HMA1 + & $1174.14 \mathrm{bB}$ & $893.0 \mathrm{cB}$ & 9046.06 & 77.95 & 9124.02 \\
& CE4. HMA2 + & $1715.6 \mathrm{aB}$ & $797.57 \mathrm{aB}$ & 3212.03 & 79.38 & 3291.41 \\
& CE8. HMA - & $1132.03 \mathrm{aC}$ & $1042.41 \mathrm{bA}$ & 4150.31 & 124.17 & 4274.49 \\
& CE8. HMA1 + & $1163.92 \mathrm{bC}$ & $887.7 \mathrm{cA}$ & 2840.36 & 64.94 & 2905.30 \\
& CE8. HMA2 + & $1100.26 \mathrm{aC}$ & $989.87 \mathrm{aA}$ & 3583.81 & 92.36 & 3676.17 \\
Segunda & T. HMA - & $982.04 \mathrm{bA}$ & $797.08 \mathrm{bC}$ & 942.30 & 15.05 & 957.35 \\
& T. HMA1 + & $2022.11 \mathrm{aA}$ & $1792.51 \mathrm{cC}$ & 1421.49 & 19.79 & 1441.29 \\
& T. HMA2 + & $1902.9 \mathrm{aA}$ & $626.95 \mathrm{aC}$ & 1535.69 & 19.54 & 1555.23 \\
& CE4. HMA - & $1617.95 \mathrm{bA}$ & $1785.04 \mathrm{bB}$ & 1265.98 & 81.48 & 1367.46 \\
CE4. HMA1 + & $1100.13 \mathrm{aA}$ & $1288.22 \mathrm{cB}$ & 938.67 & 20.09 & 958.76 \\
CE4. HMA2 + & $1753.65 \mathrm{aA}$ & $2265.08 \mathrm{aB}$ & 816.78 & 10.35 & 827.13 \\
CE8. HMA - & $895.1 \mathrm{bB}$ & $2862.54 \mathrm{bA}$ & 2132.89 & 37.55 & 2161.44 \\
CE8. HMA1 + & $1769.66 \mathrm{aB}$ & $835.64 \mathrm{cA}$ & 1282.56 & 25.61 & 1308.20 \\
CE8. HMA2 + & $1124.15 \mathrm{aB}$ & $3888.18 \mathrm{aA}$ & 1613.89 & 23.71 & 1637.60 \\
\hline
\end{tabular}

Medias sin letras o con letras iguales en la misma columna no son estadísticamente diferentes (Tukey, 0.05); las letras en minúscula representan los subgrupos formados por el factor HMA y las letras mayúsculas por el factor salinidad derivados de la prueba de Tukey, HMA- (plantas testigo); HMA1+ (aislado de Acacia sp.); HMA2+ (aislado de Larrea-Opuntia); AA: ácido ascórbico; FT: fenoles totales; DHC: dihidrocapsaicina; CT: capsaicionoides totales; PS: peso seco; CE: conductividad eléctrica; $T$ = testigo. 
con respecto a las plantas sin este tipo de estrés. Por su parte Subramanian et al. (2006) detectaron que la cantidad de este compuesto fue mayor en plantas inoculadas con $R$. intraradices en comparación con plantas sin inocular.

Fenoles totales. La concentración de fenoles totales en los frutos fue afectada por los tres factores, HMA, salinidad y cosecha. Los tratamientos inoculados con HMA2 presentaron una mayor cantidad de fenoles totales, en comparación con las plantas inoculadas con HMA1. Las plantas tratadas con salinidad presentaron mayor cantidad de fenoles con respecto a las plantas testigo. Entre las cosechas, las plantas sin inocular e inoculadas con HMA2 aumentaron significativamente la cantidad de fenoles en la segunda cosecha (Cuadro 5). Los fenoles son metabolitos secundarios que las plantas producen ante un estrés. La síntesis y acumulación de polifenoles es estimulada ante factores de estrés abiótico como la salinidad.

Estos compuestos se incrementan en diferentes tejidos de la planta, lo que aumenta la cantidad de fenoles totales en condiciones de estrés salino (Ramakrishna y Ravishankar, 2011). Se ha observado el aumento de los compuestos fenólicos ante la presencia de patógenos, como respuesta al estrés. Plantas inoculadas con HMA y con el patógeno Phytophthora capsici tuvieron un incremento en el contenido de estos compuestos comparadas con las plantas testigo (Ozgonen et al., 2009). Se puede considerar entonces que la concentración de fenoles totales incrementa como respuesta a cualquier tipo de estrés, sea biótico o abiótico.

Capsaicinoides. Solamente en la primera cosecha se registró un efecto significativo de los factores estudiados sobre la capsaicina, dihidrocapsaicina y capsaicinoides totales. Los chiles estudiados presentaron mayor cantidad de capsaicina que de dihidrocapsaicina. Además, la cantidad de estos compuestos disminuyó en la segunda cosecha (Cuadro 5). La cantidad de capsaicinoides totales concuerda con otros estudios reportados, tales como Alvarez-Parrilla et al. (2011), quienes en chile Jalapeño fresco y encontraron entre 740 y $3197 \mu \mathrm{g}$ capsaicnoides $\mathrm{g}^{-1}$ peso seco. Arrowsmith et al. (2012), concluyeron que la concentración de capsaicina en frutos de chile Jalapeño no es afectada por la aplicación de soluciones de $\mathrm{NaCl}$ a diferentes porcentajes.

\section{CONCLUSIONES}

Los consorcios de HMA aislados de las plantas nativas del Desierto Chihuahuense tuvieron un efecto diferencial en el desarrollo y rendimiento de las plantas de chile. Mientras que las plantas inoculadas con HMA1 tuvieron un menor número de frutos por planta con respecto al testigo, el peso fresco total de los frutos por planta no se vio afectado, lo que indica que los rendimientos totales no se vieron afectados por la inoculación con HMA. Los frutos de las plantas de chile inoculadas con el consorcio HMA2 mostraron la mayor concentración de $\mathrm{P}$ en la primera cosecha a pesar de la reducción en $\mathrm{P}$ exógeno aplicado, lo que demuestra el incremento en la capacidad de captación de P proporcionado por los HMA.

La salinidad tuvo un efecto negativo sobre el número de hojas, área foliar, altura, diámetro del tallo, peso fresco y peso seco, y verdor de las plantas. En general, a mayor salinidad mayor afectación de las plantas. En cuanto a los frutos, el incremento en la salinidad del agua de riego modificó significativamente el contenido de fitoquímicos y disminuyó la concentración de ácido ascórbico en éstos, pero incrementó la concentración de fenoles totales.

\section{BIBLIOGRAFÍA}

Alvarez-Parrilla E., L. A. de La Rosa, R. Amarowicz and F. Shahidi (2011) Antioxidant activity of fresh and processed Jalapeño and Serrano peppers. Journal of Agricultural and Food Chemistry 59:163173.

Arrowsmith S., T. Egan, J. F. Meekins, D. Powers and M. Metcalfe (2012) Effects of salt stress on capsaicin content, growth and fluorescence in a Jalapeño cultivar of Capsicum annuum (Solanaceae). Bios 83:1-7.

Azuma R., N. Ito, N. Nakayama, R. Suwa, N. Nguyen, J. A. Larrinaga-Mayoral, M. Esaka, H. Fujiyama and H. Saneoka (2010) Fruits are more sensitive to salinity than leaves and stems in pepper plants (Capsicum annnuum L.). Scientia Horticulturae 125:171-178.

Beltrano J., M. Ruscitti, M. C. Arango and M. Ronco (2013) Effects of arbuscular mycorrhiza inoculation on plant growth, biological and physiological parameters and mineral nutrition in pepper grown under different salinity and P levels. Journal of Soil Science and Plant Nutrition 13:123-141.

Covacevich F., H. Echeverria y L. Aguirrezabal (2001) Comparación de dos técnicas de cuantificación de infección micorritica. Ciencia del Suelo 19:155-158.

Davies Jr. F. T., V. Olalde-Portugal, M. J. Alvarado, H. M. Escamilla, R. C. Ferrera-Cerrato and J. I. Espinosa (2000) Alleviating phosphorus stress of chile ancho pepper (Capsicum annuum L. 'San Luis') by arbuscular mychorrhizal inoculation. The Journal of Horticultural Science and Biotechnology 75:655-661.

Díaz F. A., M. Alvarado C., F. Ortiz C. y 0. Grageda C. (2013) Nutrición de la planta y calidad de fruto de pimiento asociado con micorriza arbuscular en invernadero. Revista Mexicana de Ciencias Agrícolas 4:315-321.

Gerdemann J. W. and T. H. Nicolson (1963) Spores of mycorrhizal Endogone species extracted from soil by wet sieving and decanting Transactions of the British Mycological Society 46:235-244.

González-Aguilar G., R. M. Robles-Sánchez, M. A. Martínez-Téllez, G. I. Olivas, E. Álvarez-Parrilla and L. A. de la Rosa (2008) Bioactive compounds in fruits: health benefits and effect of storage conditions. Stewart Postharvest Review 4:1-10.

Jackson M. L. (1982) Determinaciones de fósforo en los suelos. In: Análisis Químico de Suelos. Editorial Omega. Barcelona, España. pp:190-253.

Kaya C., M. Ashraf, O. Sonmez, S. Aydemir, A. Levent T. and M. Ali Cullu (2009) The influence of arbuscular mycorrhizal colonisation on key growth parameters and fruit yield of pepper plants grown at high salinity. Scientia Horticulturae 121:1-6.

Khan M. H., M. K. Meghvansi, R. Gupta, V. Veer, L. Singh and M. C. Kalita (2014) Foliar spray with Vermiwash modifies the arbuscular mycorrhizal dependency and nutrient stoichiometry of Bhut Jolokia (Capsicum assamicum). PLoS ONE 9:e92318. 
Kumar A., J. F. Dames, A. Gupta, S. Sharma, J. A. Gilbert and P. Ahmad (2015) Current developments in arbuscular mycorrhizal fungi research and its role in salinity stress alleviation: a biotechnological perspective. Critical Reviews in Biotechnology 35:461-474.

Niu G., D. S. Rodríguez, E. Call, P. W. Bosland, A. Ulery and E. Acosta (2010) Responses of eight chile peppers to saline water irrigation. Scientia Horticulturae 126:215-222.

Niu G., P. Osuna, Y. Sun and S. Rodriguez (2012) Seedling emergence, growth, and mineral nutrition of ornamental chile peppers irrigated with saline water. HortScience 47:1653-1657.

Oboh G. and J. B. T. Rocha (2007) Polyphenols in red pepper [Capsicum annuum var. aviculare (Tepin) and their protective effect on some pro-oxidants induced lipid peroxidation in brain and liver. European Food Research and Technology 225:239-247.

Ozgonen H., N. Yardimci and H. Culal Kilic (2009) Induction of Phenolic compounds and pathogenesis - related proteins by mycorrhizal fungal inoculations against Phytophthora capsici Leonian in Pepper. Pakistan Journal of Biological Sciences 12:1181-1187.

Phillips J. M. and D.S. Hayman (1970) Improved procedures for clearing roots and staining parasitic and vesicular-arbuscular mycorrhizal fungi for rapid assessment of infection. Transactions of the
British Mycological Society 55:158-161.

Ramakrishna A. and G. A. Ravishankar (2011) Influence of abiotic stress signals on secondary metabolites in plants. Plant Signaling \& Behavior 6:1720-1731.

Selvakumar G. and P. Thamizhiniyan (2011) The effect of the arbuscular mycorrhizal (AM) fungus Glomus intraradices on the growth and yield of chilli (Capsicum annuum L.) under salinity stress. World Applied Sciences Journal 14:1209-1214

Sharif M. and N. Claassen (2011) Action mechanisms of arbuscular mycorrhizal Fungi in phosphorus uptake by Capsicum annuum L. Pedosphere 21:502-511.

SIAP, Servicio de Información Agroalimentaria y Pesquera (2015) Anuario estadístico de producción agrícola. http://infosiap.siap.gob.mx/ aagricola_siap_gb/icultivo/index.jsp (Marzo 2017)

Smith S. E. and D. Read (2008) The symbionts forming arbuscular mycorrhizas. In: Mycorrhizal Symbiosis. Elsevier. Great Britain. pp:1341.

Subramanian K. S., P. Santhanakrishnan and P. Balasubramanian (2006) Responses of field grown tomato plants to arbuscular mycorrhizal fungal colonization under varying intensities of drought stress. Scientia Horticulturae 107:245-253. 
